\title{
PODER RELATIVO DO LUCRO CONTÁBIL E DO FLUXO DE CAIXA DAS OPERAÇÕES PARA PREVER FLUXOS DE CAIXA FUTUROS: UM ESTUDO EMPÍRICO NO BRASIL
}

\author{
RELATIVE POWER OF THE ACCOUNTING PROFIT AND OF THE CASH \\ FLOW FROM FUTURE CASH FLOWS PREDICTION OPERATIONS: \\ EMPIRIC STUDY IN BRAZIL
}

\section{PODER RELATIVO DEL LOGRO CONTABLE Y DEL FLUJO DE CAJA DE LAS OPERACIONES PARA PREVER FLUJOS DE CAJA FUTUROS: UN ESTUDIO EMPÍRICO EN BRASIL}

\author{
DR. PAULO ROBERTO B. LUSTOSA \\ Professor Titular, Universidade de Brasília \\ prblustosa@gmail.com \\ DR. ARIOVALDO DOS SANTOS \\ Professor Titular, Universidade de São Paulo \\ arisanto@usp.br
}

\section{RESUMO}

Este artigo avalia a eficácia da previsão de fluxos futuros, para um e dois anos à frente, de quatro medidas de desempenho da empresa, sendo duas de fluxo de caixa e duas de lucro, a partir de medidas ex post do Fluxo de Caixa das Operações (FCO), isolado e em conjunto com a variação das alocações (accruals) de curto prazo $\left(\Delta \mathrm{Ac}_{\mathrm{cp}}\right)$, e do Lucro Líquido contábil (LL). A metodologia de previsão é do tipo externo à amostra. São feitas várias previsões anuais e bianuais transversais para as 92 empresas que compõem a amostra, ao longo dos anos de 1996 a 2004. Os desvios (erros) médios absolutos de previsão, a Mediana dos erros e as correlações médias entre os valores reais e projetados das variáveis são apurados e comparados entre si para fins de verificação da eficácia de previsão dos fluxos futuros de cada um dos três modelos. Os resultados revelam que: (1) LL é superior ao FCO para prever o Lucro Líquido do ano se- 
guinte, mas, ao contrário, FCO é superior ao LL para prever o Lucro Líquido dois anos à frente; (2) a combinação das alocações de curto prazo $\left(\Delta A c_{c p}\right)$ com o $F C O$ não melhora as previsões que são feitas apenas com o FCO, indicando que o Fluxo de Caixa das Operações é mais eficaz para prever fluxos futuros de caixa e de lucro do que o capital circulante líquido; e (3) o Lucro Operacional (LO), como é definido na legislação societária brasileira, é de difícil previsão.

Palavras-Chave: Previsão de Fluxos Futuros. Regime de Competência. Regime de Caixa. Modelos Econométricos.

\section{ABSTRACT}

This paper evaluates the accuracy of three accounting performance measures (net income - NI, cash flows from operations - CFO, and working capital - WC) for predicting two cash flows (cash flows from operations - CFO, and free cash flows - FCF) and two income metrics (net income - NI, and operating earnings - OE), one and two years in the future. We use out-of-sample regressions for forecasting the above metrics during the time-period from 1996 to 2004 . The prediction accuracy of each forecasted variable is evaluated by computing three measures: the mean absolute errors of the forecasted and actual amounts series; the median of the prediction errors; and the squared correlation $\left(\mathrm{R}^{2}\right)$ between the forecasted and the actual amounts. The findings show that: (1) NI is superior to CFO for predicting $\mathrm{NI}$ one year in the future, but CFO overcomes $\mathrm{NI}$ when the prediction of $\mathrm{NI}$ is for two years in the future; (2) WC does not beat CFO for predicting neither one of the four performance measures one and two years ahead; and (3) operating earnings (OE), as defined in the Brazilian Corporate Law, are difficult to predict.

Keywords: Prediction of Future Flows. Accrual Accounting. Cash-Basis Accounting. Econometric Models.

\section{RESUMEN}

Este artículo evalúa la eficacia de la previsión de flujos futuros, para uno y dos años más adelante, de cuatro medidas de desempeño de la empresa, siendo dos de flujo de caja y dos de beneficio, desde medidas ex post del Flujo de Caja de las Operaciones (FCO), separado y en conjunto con la variación de las ubicaciones (accruals) de corto plazo ( $\triangle \mathrm{AcB}$ $\mathrm{cpB}$ ), y del Beneficio Líquido contable (LL). La metodología de previsión es del tipo externo a la muestra. Son hechas varias previsiones anuales y bianuales transversales para las 92 empresas que componen la muestra, a lo largo de los años de 1996 a 2004. Los desvíos (errores) medios absolutos de previsión, la Mediana de los errores y las correlaciones me- 
dias entre los valores reales y proyectados de las variables son apurados y comparados entre sí para fines de verificación de la eficacia de previsión de los flujos futuros de cada un0 de los tres modelos. Los resultados revelan que: (1) LL es superior al FCO para prever el Beneficio Líquido del año siguiente, pero, al contrario, FCO es superior al LL para prever el Beneficio Líquido dos años hacia adelante; (2) la combinación de las ubicaciones de corto plazo $(\triangle \mathrm{AcB} c \mathrm{~B})$ con el FCO no mejoran las previsiones que son hechas apenas con el FCO, indicando que el Flujo de Caja de las Operaciones es más eficaz para prever flujos futuros de caja y de beneficio que el capital circulante líquido; y (3) el Beneficio Operacional (LEO), como es definido en la legislación societaria brasileña, es de difícil previsión.

Palabras-Clave: Previsión de Flujos Futuros. Régimen de Capacidad. Régimen de Caja. Modelos Econométricos.

\section{INTRODUÇÃO}

O objetivo principal das demonstrações financeiras, segundo o Financial Accounting Standards Board (FASB), é permitir aos usuários estabelecer previsões do volume, momento de ocorrência e risco associado aos Fluxos de Caixa Futuros da empresa (Statement of Financial Concept - SFAC 1, 1978, p. 5). Uma vez que o efeito das decisões sempre ocorre no futuro, investidores, credores, gestores, etc. decidem com base em expectativas sobre os Fluxos de Caixa Futuros da empresa, em termos amplos, ou do ativo objeto da decisão em termos restritos. Para tanto, vale-se de informações que os ajudem nessas previsões, sendo as demonstrações financeiras um elemento importante nesse processo.

O FASB assume, formalmente, no SFAC 1 (1978, par. 48), que o Lucro Contábil é uma medida mais relevante do que o Fluxo de Caixa das Operações para prever os Fluxos de Caixa Futuros da empresa. A racionalidade que dá suporte a esse posicionamento parece derivar das várias estimativas que os contadores fazem para mensurar o

A palavra accruals costuma ser utilizada na língua inglesa para designar o modelo de contabilidade pelo regime de competência (accrual-basis accounting) em oposição à contabilidade pelo regime de caixa (cash-basis accounting). Essa palavra deriva do verbo to accrue, que significa agrupar ou ajuntar coisas. Portanto, mesmo na língua inglesa, o uso da palavra accrual em contabilidade parece ter um significado ligeiramente diferente da palavra raiz da qual ela deriva. Em substância, accrual deveria relacionar-se a todas as alocações de receitas e despesas feitas ao lucro, em momentos defasados do efeito no caixa. Não contemplaria, nesse sentido, os recebimentos e pagamentos associados a receitas e despesas antes reconhecidas. Na prática, contudo, accrual tem sido utilizada em um sentido ligeiramente diferente, designando as diferenças entre o lucro e o Fluxo de Caixa das Operações de um mesmo período. Ante a inexistência de uma única palavra em português para designar o sentido que o termo em inglês designa, optou-se por utilizar a palavra original estrangeira neste artigo. 
lucro pelo regime de competência, chamados genericamente na literatura estrangeira de accruals ${ }^{1}$. Entretanto, não há evidências empíricas definitivas que corroborem a afirmação do FASB.

O uso de diferentes abordagens metodológicas e a forma como são definidas as variáveis pode ser uma das razões para a não-existência de consenso entre os pesquisadores sobre a superioridade relativa do lucro e do fluxo de caixa para prever os Fluxos de Caixa Futuros. Por exemplo, Finger (1994), utilizando uma longa série temporal de 50 empresas e com metodologia de projeção do futuro externa à amostra, concluiu que o Fluxo de Caixa das Operações é marginalmente superior ao Lucro Contábil nas previsões dos fluxos de caixa de curto prazo, porém, o poder preditivo dessas duas variáveis é equivalente quando o horizonte de projeção é aumentado. Contudo, Nichols e Wahlen (2004) concluíram que "as variações anuais do Lucro Contábil afetam muito mais substancialmente o retorno das ações do que o Fluxo de Caixa das Operações." Mais recentemente, ainda, pesquisa de Lev et al. (2005), utilizando a mesma técnica de projeção externa à amostra para períodos anuais usada por Finger (1994), revelou que o Fluxo de Caixa das Operações supera o Lucro Contábil ou se equivale a este nas projeções de quatro variáveis (Fluxo de Caixa das Operações, Fluxo de Caixa Livre, Lucro Operacional e Lucro Líquido) para períodos futuros de um ano, dois anos e dois e três anos acumulados.

A relevância do tema e o fato de ainda ser esta uma questão que permanece em aberto motivam a realização desse tipo de análise empírica no Brasil. O objetivo, portanto, deste trabalho é avaliar a eficácia da projeção ex ante de quatro medidas futuras de fluxo de caixa (serão detalhadas na seção de metodologia) para um e dois anos à frente, a partir de medidas ex post do Fluxo de Caixa das Operações (FCO), isolado e em conjunto com os accruals de curto prazo e do Lucro Líquido contábil.

Optou-se pela metodologia de projeção ex ante dos fluxos futuros porque esta é a única que pode ser utilizada em situações concretas do mundo real, uma vez que as decisões são tomadas em um ambiente de incertezas sobre o futuro. Nesse tipo de metodologia, denominada externa à amostra (out of sample), projeta-se período a período o valor da variável de todas as empresas (cross-section) para os períodos futuros desejados. Ela contrasta com um outro tipo de abordagem, ainda muito comum na literatura, em que o futuro é projetado a partir de parâmetros únicos obtidos para toda a série temporal (in-sample), que traz implícita a premissa, bastante discutível, de comportamento regular das variáveis sob estudo ao longo de todo o período [ver a respeito os estudos de Poon e Granger (2003) e Goyal e Welch (2004)].

Na continuação deste artigo, a seção dois apresenta a teoria e uma breve revisão da literatura sobre o ponto que será investigado; a seção três detalha a amostra e a metodologia da pesquisa; a seção quatro analisa os resultados; e a seção cinco conclui. Ao final, são apresentadas as referências consultadas. 


\section{repec}

Poder Relativo do Lucro Contábil e do Fluxo de Caixa das operações para prever Fluxos de Caixa Futuros: um Estudo Empírico no Brasil

\section{TEORIA E REVISÃO DA LITERATURA}

A questão subjacente desta pesquisa é muito polêmica, uma vez que toca o centro do modelo normativo de contabilidade financeira adotado em todo o mundo: qual é a eficácia do Lucro Contábil, apurado pelas regras do regime de competência, para prever os Fluxos de Caixa Futuros da empresa, relativamente aos fluxos de caixa passados? Em outras palavras, qual variável tem maior poder preditivo dos Fluxos de Caixa Futuros, o lucro ou o próprio fluxo de caixa corrente das operações, sozinho e em conjunto com as alocações de curto prazo?

Sabe-se que todos os critérios de apuração do lucro, os conhecidos ou os que ainda vierem a ser estabelecidos, produzem lucros periódicos diferentes. Contudo, o verdadeiro lucro, na perspectiva dos donos do capital, é uma diferença aritmética, ajustada no tempo pela inflação e pelo custo de oportunidade do capital para uma mesma data focal, entre todo o dinheiro que entrou e que saiu do empreendimento, o que só pode ser conhecido quando a entidade termina e baixa todos os seus ativos (Martins, 2001). Entretanto, razões de ordem prática impõem a apuração do lucro em intervalos menores, resultando em valores diferentes, conforme sejam os conceitos adotados para mensurá-lo.

Ao realizar transações econômicas, os agentes se interessam pelos benefícios líquidos futuros que os ativos obtidos lhes gerarão. Após a decisão, o ativo sacrificado na troca não é mais relevante para quem o sacrificou, mas apenas para quem o recebeu. Os benefícios podem ser explicitados em unidades monetárias. Logo, a medida relevante é quanto vale um ativo e não quanto ele custou. O lucro periódico correto de uma empresa (um agregado de ativos) seria, portanto, a diferença do valor da empresa entre os momentos extremos desse período (Guerreiro, 1989). Ante o dilema da subjetividade para se mensurar o valor dos ativos, a contabilidade estruturou-se sobre o conceito mais objetivo e confiável de lucro realizado, aquele que já foi transformado em dinheiro ou está tão próximo dessa transformação que já pode ser considerado dinheiro.

Mas mesmo a opção pela objetividade conservadora tem muitos aspectos subjetivos. Os ativos reconhecidos por seus custos não-expirados são alocados ao resultado, para confrontação com as receitas realizadas, proporcionalmente ao seu consumo estimado. Esse processo e as demais provisões ativas e passivas presentes no regime de competência dão margem a alocações arbitrárias de despesas e receitas ao resultado, tornando o Lucro Contábil uma medida gerenciável (Ijiri, 2002). Cria-se, assim, uma diferença temporal entre o lucro e o Fluxo de Caixa das Operações, que resulta de todas as despesas (receitas) ainda não-pagas (recebidas) que são alocadas ao lucro do período. Tais alocações, denominadas genericamente na literatura de língua inglesa por accruals, são estimativas e por isso geram dúvidas nos usuários dos relatórios contábeis, sobre a qualidade do Lucro Contábil divulgado. 
Visando suprir o usuário com uma medida mais confiável do desempenho da empresa, complementar ao lucro, o FASB exigiu, ainda na segunda metade da década de 1980, que as empresas divulgassem também um relatório mostrando o seu fluxo de caixa do mesmo período a que se refere o lucro. Esse relatório desdobra o fluxo de caixa total em três grupos de atividades, sendo o primeiro deles o Fluxo de Caixa das Operações (FCO), uma medida aproximada do lucro da empresa por regime de caixa. Temendo que o FCO pudesse vir a substituir o lucro nas análises da empresa pelos investidores, o FASB (SFAC 1, 1978, par. 44), ainda antes de tornar a demonstração dos fluxos de caixa obrigatória, estabeleceu, na estrutura conceitual de suas normas, que: “... em geral o Lucro Contábil é um melhor indicador para previsão da potencialidade de a empresa gerar fluxos contínuos e favoráveis de caixa do que as informações de recebimento e pagamento de caixa (passados)." Além disso, o SFAS-95 - Statement of Cash Flows, de 1987, proíbe a divulgação de informações do fluxo de caixa por ação.

Alguns estudos empíricos sobre o poder informativo para o mercado do lucro e do fluxo de caixa e, por extensão, dos accruals de curto e longo prazos são anteriores ao SFAS-95, mas houve uma intensificação desse tipo de estudo após essa norma, devido, entre outras razões, à formação do banco de dados que a norma propiciara. Um dos primeiros estudos nessa linha foi o de Wilson (1986), que documenta respostas diferenciadas do mercado aos accruals de curto e longo prazos. No mesmo ano, o estudo de Rayburn et al. (1986) fornece evidência de poder informativo incremental dos accruals além do FCO. Na seqüência, vieram várias outras pesquisas, com pequenas variações em torno desse tema central e com alguns refinamentos metodológicos, merecendo destaque Bowen et al. (1987), sugerindo que o FCO tem associação mais forte com o retorno das ações do que com os accruals, enquanto que Bernard e Stober (1989) observam que o impacto do FCO e dos accruals no preço das ações depende de vários outros fatores, incluindo as condições econômicas e a qualidade da mensuração das variáveis.

Na década de 90, seguindo as observações de Bernard e Stober (1989), as pesquisas sobre esse tema passaram a focar também elementos relacionados às propriedades do Lucro Contábil como aspectos de sua persistência e desdobramentos dos seus componentes. São exemplos os estudos de Ali (1994), Sloan (1996), Dechow (1996) e Pfeiffer et al. (1998).

Nos anos 2000, houve uma retomada da linha de estudos iniciada por Finger (1994), em que o foco de interesse se desloca para a relação do lucro do FCO e do accruals com os Fluxos de Caixa Futuros diretamente, e não mais com o preço das ações. Os fluxos futuros de caixa são medidas contábeis de desempenho já observadas como formas de Lucro Contábil (Lucro Líquido e Lucro Operacional) e de fluxo de caixa (Fluxo de Caixa das Operações e Fluxo de Caixa Livre), que são comparados com antecipações dessas mesmas variáveis a partir do Lucro Contábil, do Fluxo de Caixa das Operações e dos vários tipos de accruals. A 
qualidade da predição, nesse caso, é aferida pelos erros médios e Medianas entre os valores projetados e os valores reais das variáveis preditas. Merecem destaque, nesse sentido, os estudos de Barth et al. (2001) e Lev et al. (2005). É interessante observar que esse último estudo conclui, utilizando duas metodologias diferentes aplicadas sobre uma amostra de mais de trinta mil observações, que o FCO, sozinho, supera o Lucro Contábil e que o FCO combinado a accruals na previsão de Fluxos de Caixa Futuros e lucros contábeis em quase todos os vários horizontes de projeção utilizados no estudo.

No Brasil, aos poucos vai se formando um corpo de pesquisas empíricas em contabilidade, mas não se verificou nenhum estudo com foco específico no ponto que é investigado neste artigo. Contudo, já é considerável o número de estudos que tratam do impacto de variáveis contábeis no preço das ações, o que, indiretamente, tem relação com o tema aqui abordado, já que o preço das ações reflete uma expectativa do mercado sobre os Fluxos de Caixa Futuros das empresas. São exemplos de pesquisas nessa linha, utilizando dados de empresas localizadas no Brasil, os trabalhos de Lopes (2001), que documenta uma maior relação entre o patrimônio líquido e o preço das ações, Martinez (2002), que encontra evidências de manipulação de accruals para gerenciamento do resultado; e Dantas (2005), que conclui pela existência de relação significativa entre o grau de alavancagem operacional e o retorno das ações. Por outro lado, Lustosa (2001), utilizando metodologia de simulação aleatória de dados contábeis, constata que a relação do Lucro Contábil e do Fluxo de Caixa das Operações com os Fluxos de Caixa Futuros da empresa depende da idade dos investimentos já realizados e dos planos ainda não-implementados, o que, de certa forma, corrobora os achados empíricos do estudo de Bernard e Stober (1989).

\section{METODOLOGIA}

\section{Amostra}

A amostra para realizar esta pesquisa é composta de 92 empresas de capital aberto, não-financeiras, listadas na Bolsa de Valores do Estado de São Paulo (Bovespa). Foram coletados dados anuais das variáveis necessárias, no banco de dados da Economática, de 1996 a 2004. Para cada uma das cinco variáveis finais da pesquisa, conforme detalhado na subseção seguinte, foi obtido um painel com 828 observações (92 empresas x 9 anos), resultando em um painel final com 4.140 observações.

\section{Modelos de Previsão}

Os Fluxos de Caixa Futuros serão representados por duas variáveis que são diretamente fluxos de caixa (FCO = Fluxo de Caixa das Operações; e FCL = Fluxo de Caixa Livre) e duas variáveis que representam fluxos de caixa indiretamente ( $L L$ = Lucro Líquido; e LO = Lucro Operacional). Embora sejam as mesmas variáveis utilizadas no estudo de Lev 
et al. (2005), a definição destas é ligeiramente diferente. Aqueles autores utilizaram o FCO fornecido pelas empresas, uma vez que nos Estados Unidos, onde foi realizada a pesquisa, a demonstração de fluxos de caixa é obrigatória; neste estudo, FCO teve que ser apurado pelo método indireto, ajustando-se o lucro pelos seus principais accruals. A variável $\mathrm{FCL}$ aqui é definida como FCO menos as despesas de depreciação e amortização do período. A racionalidade para isto é que se está considerando livre apenas o dinheiro que sobra das operações após os desembolsos para recuperar os investimentos consumidos. No estudo de Lev et al. (2005), FCL foi definido como FCO menos todos os desembolsos em novos investimentos do período, e não somente a recuperação dos investimentos consumidos. LL neste estudo é a última linha da demonstração do resultado; naquele é o lucro antes do imposto de renda e dos itens extraordinários. Finalmente, LO aqui inclui as despesas financeiras e o resultado da equivalência patrimonial, como definido na Lei $n^{\circ} 6.404 / 76$, enquanto que no estudo de Lev et al. (2005) esses itens são excluídos.

As quatro variáveis foram previstas, ex ante, ano a ano e transversalmente, para um ano e dois anos à frente, conforme os exemplos a seguir:

A) Previsão de $\mathrm{FCL}$ para o próximo ano $\left(\mathrm{FCL}_{\mathrm{t}+1}\right)$

a) Previsão a partir de FCO (exemplo para 1999):

- Estimativa transversal: $\mathrm{FCL}(98)=\alpha+\beta \mathrm{FCO}(97)+\varepsilon$

- Previsão: $E[F C L(99)]=\alpha+\beta F C O(98)$, utilizando os parâmetros $\alpha$ e $\beta$ obtidos conforme acima.

- Erro de previsão: $\mathrm{FCL}(99)$ - $\mathrm{E}[\mathrm{FCL}(99)]$.

Aqui se prevê FCL para o ano de 1999 a partir do FCO corrente de 1998. Em primeiro lugar, é rodada uma regressão transversal do FCL real de 1998 contra o FCO real de 1997, a fim de obter-se os parâmetros $\alpha$ e $\beta$. Em seguida, esses parâmetros são aplicados aos valores correntes de FCO de 1998, gerando a previsão de FCL para 1999. Finalmente, o FCL projetado para 1999 é comparado com o FCL real desse ano, obtendo-se o erro de previsão. O mesmo procedimento é repetido para todos os anos.

b) Previsão a partir de $\mathrm{FCO}$ e dos accruals de curto prazo: $\triangle \mathrm{Ac} \mathrm{c}_{\mathrm{cp}}$ (estamos definindo os accruals de curto prazo como a variação de todas as contas operacionais do ativo $\mathrm{e}$ passivo circulantes, exceto a conta de disponibilidades)

- Estimativa transversal: $\mathrm{FCL}(98)=\alpha+\beta_{1} \mathrm{FCO}(97)+\beta_{2} \Delta \mathrm{Ac}_{\mathrm{cp}}(97)+\varepsilon$

- Previsão: $\mathrm{E}[\mathrm{FCL}(99)]=\alpha+\beta_{1} \mathrm{FCO}(98)+\beta_{2} \Delta \mathrm{Ac}_{\mathrm{cp}}$ (98), utilizando os parâmetros $\alpha$, $\beta_{1}$ e $\beta_{2}$ obtidos conforme acima.

- Erro de previsão: conforme em "a".

O mesmo procedimento utilizado para prever $\mathrm{FCL}$ é repetido para a previsão de FCO, LO e LL. 
c) Previsão a partir do Lucro Líquido (LL) - exemplo para o ano de 1999:

- Estimativa transversal: $\mathrm{FCL}(98)=\alpha+\beta L L(97)+\varepsilon$

- Previsão: $E[F C L(99)]=\alpha+\beta L L(98)$, utilizando os parâmetros $\alpha$ e $\beta$ obtidos conforme acima.

- $\quad$ Erro de previsão: FCL(99) - E[FCL(99)].

Aqui a variável independente para prever a variável dependente FCL é o Lucro Líquido (LL) e não mais o FCO. Afinal, o objetivo deste trabalho é avaliar qual variável é mais eficaz para prever os Fluxos de Caixa Futuros no Brasil: se é o Fluxo de Caixa das Operações (FCO), isolado ou em conjunto com os accruals de curto prazo $\left(\triangle A c_{c p}\right)$, ou se é o Lucro Líquido (LL). Observe que quando se combina o FCO com os accruals de curto prazo obtém-se uma medida equivalente ao capital circulante líquido gerado pelas operações, uma espécie de caixa bruto, uma vez que nessa medida ainda estão presentes os accruals de curto prazo. O Lucro Líquido (LL) é uma medida ainda mais grosseira de Fluxo de Caixa das Operações, pois contém também os accruals mais problemáticos de longo prazo, embora estes não estejam sendo considerados de modo desagregado neste trabalho.

B) Previsão de $\mathrm{FCL}$ para dois anos à frente $\left(\mathrm{FCL}_{\mathrm{t}+2}\right)$

d) Previsão a partir de FCO (exemplo para o ano 2000):

- Estimativa transversal: $\mathrm{FCL}(98)=\alpha+\beta \mathrm{FCO}(96)+\varepsilon$

- Previsão: $E[F C L(00)]=\alpha+\beta F C O(98)$, utilizando os parâmetros $\alpha$ e $\beta$ obtidos conforme acima.

- Erro de previsão: FCL(00) - E[FCL(00)].

Aqui se repete a mesma lógica do item A), com um intervalo de dois anos para a estimativa dos parâmetros e para o modelo de previsão. Inicialmente, o FCL do ano de 1998 é estimado regredindo-se este contra o FCO do ano de 1996. Obtém-se, assim, os parâmetros $\alpha$ e $\beta$ que são utilizados para fazer a previsão do FCL para o ano 2000. Finalmente, apura-se o erro de previsão, pela comparação do FCL projetado para 2000 com o FCL real desse ano. O mesmo procedimento é repetido para as demais variáveis dependentes, isto é, FCO, LO e LL.

e) Previsão a partir de FCO e dos accruals de curto prazo: $\triangle \mathrm{Ac}_{\mathrm{cp}}$ (estamos definindo os accruals de curto prazo como a variação de todas as contas operacionais do Ativo e Passivo Circulantes, exceto a conta de disponibilidades) regressões, nos casos em que existem covariâncias importantes entre o deflator escolhido e a variável dependente. Não é o caso da presente pesquisa, posto que o mesmo deflator está presente em ambos os lados da equação de regressão. Para aprofundamento nos problemas que o ajuste do efeito tamanho pode causar no resultado das regressões, ver Lubberink e Pope (2005). 
- Estimativa transversal: $\mathrm{FCL}(98)=\alpha+\beta_{1} \mathrm{FCO}(96)+\beta_{2} \Delta \mathrm{Ac}_{\mathrm{cp}}(96)+\varepsilon$

- Previsão: $\mathrm{E}[\mathrm{FCL}(00)]=\alpha+\beta_{1} \mathrm{FCO}(98)+\beta_{2} \Delta \mathrm{Ac}_{\mathrm{cp}}(98)$, utilizando os parâmetros $\alpha$, $\beta_{1}$ e $\beta_{2}$ obtidos conforme acima.

- Erro de previsão: conforme em "d".

Inicialmente, estima-se o FCL do ano de 1998 a partir do FCO e da variação dos accruals de curto prazo $\left(\triangle \mathrm{Ac}_{\mathrm{cp}}\right)$ do ano de 1996, a fim de obter-se os parâmetros $\alpha, \beta_{1}$ e $\beta_{2}$. Depois, esses mesmos parâmetros são aplicados ao $\mathrm{FCO}$ e à $\triangle A c_{c p}$ correntes do ano de 1998, para cada uma das 92 empresas, obtendo-se o FCL projetado para o ano 2000. Finalmente, é apurado o erro de previsão, comparando-se o FCL real do ano $2000 \mathrm{com}$ o que fora projetado, dois anos antes, para esse ano. O mesmo procedimento é repetido na previsão das outras três variáveis dependentes, isto é, FCO, LO e LL.

f) Previsão a partir do Lucro Líquido (LL) - exemplo para o ano 2000:

- Estimativa transversal: $\mathrm{FCL}(98)=\alpha+\beta L L(96)+\varepsilon$

- Previsão: $E[F C L(00)]=\alpha+\beta L L(98)$, utilizando os parâmetros $\alpha$ e $\beta$ obtidos conforme acima.

- Erro de previsão: $\mathrm{FCL}(00)$ - $\mathrm{E}[\mathrm{FCL}(00)]$.

Aqui a variável independente para prever FCL passou a ser o Lucro Líquido, e não mais o FCO. Todos os demais procedimentos são similares ao que está descrito em "d".

\section{Outros aspectos metodológicos}

Todas as variáveis, as dependentes e as independentes nas regressões, são divididas pelo saldo do ativo total de cada empresa, verificado ao final dos respectivos anos, para correção do efeito tamanho das empresas sobre o valor absoluto das variáveis ${ }^{2}$. Uma revisão interessante da literatura sobre o ajuste do efeito tamanho pode ser encontrada em Bujaki e Richardson (1997).

As projeções para cada período, anual ou bianual, como visto, são feitas por meio de um procedimento externo à amostra (out of sample). Esta é a situação possível no mundo real, em que as únicas informações disponíveis são as do momento corrente e passadas. Por isso, ao invés de utilizar parâmetros (os coeficientes $\alpha$ e $\beta$ dos modelos de estimação) únicos para toda a amostra, como é comum nos modelos de projeção internos à amostra (in-sample), optou-se por rodar novas regressões transversais a cada período, obtendo-se, com isso, parâmetros diferentes em todos os períodos de projeção, que passavam a incorporar as novas informações do ano corrente.

O teste final será feito por comparação entre os erros de previsão dos três modelos ( $\mathrm{FCO}$ isolado, $\mathrm{LL}$ isolado e $\mathrm{FCO}+\Delta \mathrm{Ac}_{\mathrm{cp}}$ ) para projetar as quatro variáveis de fluxos futuros (FCO, FCL, LL e LO) um e dois anos à frente. $O$ erro de previsão será medido pelo Desvio Médio Absoluto (DMA) entre o valor previsto e o valor real da respectiva variável. O DMA é a 
média aritmética dos desvios simples absolutos de cada previsão, por empresa e por período, em relação à média geral das previsões. A comparação dos desvios será feita por meio de testes $t$ de média de amostras emparelhadas, tendo sempre o FCO isolado como referência. Os erros (desvios) serão dispostos em séries empilhadas por períodos (pooled-data), para as projeções de um e dois anos de cada uma das quatro variáveis de fluxos futuros. Além do DMA, serão apuradas ainda a Mediana (MED) da série de desvios de cada previsão e o Coeficiente de Determinação $\left(R^{2}\right)$ entre os valores previstos e reais das variáveis preditas.

\section{RESULTADOS}

\section{Estatísticas Descritivas}

A Tabela 1 a seguir mostra as principais estatísticas descritivas das variáveis desta pesquisa.

Tabela 1 - Estatísticas Descritivas

\begin{tabular}{lcccccc}
\hline Variável & Média & $\begin{array}{c}\text { Desvio } \\
\text { Padrão }\end{array}$ & Mediana & Máximo & Mínimo & Obs \\
\hline LL & 0,0009 & 0,2313 & 0,0297 & 0,8904 & $-3,2740$ & 828 \\
LO & 0,2991 & 2,5004 & 0,0233 & 39,1388 & $-3,8799$ & 828 \\
FCO & 0,0914 & 0,2652 & 0,0778 & 2,5887 & $-1,6811$ & 828 \\
FCL & 0,0110 & 0,1946 & 0,0208 & 0,6694 & $-1,7498$ & 828 \\
$\square \mathrm{Ac}_{\mathrm{cp}}$ & 0,0369 & 0,1548 & 0,0205 & 1,1962 & $-0,9197$ & 828 \\
\hline
\end{tabular}

$\mathrm{LL}=$ Lucro Líquido anual (última linha da demonstração do resultado);

LO = Lucro Operacional, conforme Lei 6.404/76, ou seja, inclui as despesas financeiras e o resultado de equivalência patrimonial;

$\mathrm{FCO}=$ fluxo de caixa das atividades operacionais. Esta variável não é fornecida diretamente, uma vez que a Demonstração dos Fluxos de Caixa ainda não é obrigatória no Brasil. Ela foi obtida adicionando-se a variação anual das contas operacionais do Ativo e Passivo Circulantes (exceto disponibilidades) ao Capital Circulante Líquido Gerado pelas Operações (CCLop), fornecido no primeiro bloco de informações da Demonstração das Origens e Aplicações de Recursos (DOAR);

$\mathrm{FCL}=$ Fluxo de Caixa Livre, obtido deduzindo-se do FCO as despesas anuais de depreciação, amortização e exaustão do período;

$\Delta \mathrm{Ac}_{\mathrm{cp}}=$ variação anual dos accruals de curto prazo, que correspondem às variações das contas operacionais do Ativo e Passivo Circulantes, exceto as disponibilidades;

Todas as variáveis acima são fluxos anuais. Os valores dessas variáveis, de cada uma das 


\section{repc}

empresas da amostra, foram divididos pelo ativo total do fim de cada ano, para correção do efeito tamanho das empresas.

De todas as variáveis, o Lucro Operacional (LO) é de longe a que apresenta maior amplitude (diferença entre os valores máximo e mínimo), como está refletido também em um desvio padrão $(2,5004) 8,36$ vezes maior do que a sua própria média. Isto, provavelmente, se deve à grande volatilidade do câmbio no período, o que afetou erraticamente as despesas financeiras de muitas empresas da amostra com passivos denominados em moeda estrangeira. A variável que apresenta menor variabilidade é a variação dos accruals de curto prazo, o que, de certa forma, mostra que as alocações ao lucro oriundas do Ativo e Passivo Circulantes são mais estáveis, deixando menor margem aos gestores para gerenciamento arbitrário do lucro usando esse tipo de accrual (LEV; LI; SOUGIANNIS, 2005). A alta variabilidade do Lucro Operacional não se transferiu, curiosamente, para o Lucro Líquido. Uma possível explicação para isto é que, na média, o resultado não-operacional e a constituição e reversão dos créditos tributários de imposto de renda e contribuição social sobre o Lucro Líquido podem ter compensado a alta variabilidade do Lucro Operacional.

A variabilidade do Fluxo de Caixa das Operações (FCO) $(0,2652)$ é ligeiramente maior do que a do Lucro Líquido (LL) (0,2313). O fluxo de caixa como um todo apresenta uma variabilidade muito maior do que a do lucro, uma vez que uma das características do regime de competência é dissolver, no tempo, os desembolsos concentrados de caixa que ocorrem nas decisões de investimento. Mas a variabilidade apenas do FCO tende a acompanhar à do Lucro Líquido, já que as receitas e as despesas reconhecidas no lucro no período $t$, mas que só sensibilizarão o $\mathrm{FCO}$ em $t+1$, são, de certa forma, compensadas com as receitas e as despesas reconhecidas no lucro em $t-1$, mas cujos efeitos no FCO ocorrem somente em $t$. A variabilidade dos Fluxos de Caixa Livres (FCL) $(0,1946)$ é menor do que a de FCO porque as despesas de depreciação, amortização e exaustão que são deduzidas de FCO para a obtenção de FCL têm um comportamento estável, já que no Brasil há uma tendência de as empresas adotarem as regras de depreciação fiscais também na contabilidade societária.

As cinco variáveis apresentam padrões de assimetria alternados, como se pode ver pela Mediana maior que a média em duas variáveis ( $L L$ e FCL), indicando assimetria negativa; e Mediana menor que a média em três variáveis ( $L O, F C O$ e $\left.\Delta A c_{c p}\right)$, indicando assimetria positiva. As premissas de normalidade, no entanto, não foram violadas, conforme testes realizados, a não ser para a variável Lucro Operacional (LO), mas optou-se por não aplicar corretivos à série de LO porque esta variável não apresentou resultados relevantes nos testes, como será visto adiante. 
Poder Relativo do Lucro Contábil e do Fluxo de Caixa das operações para prever Fluxos de Caixa

Futuros: um Estudo Empírico no Brasil

\section{Matriz de Correlações}

A Tabela 2 mostra as correlações de Pearson entre todas as variáveis.

Tabela 2 - Matriz de Correlações

\begin{tabular}{lccccc}
\hline \multicolumn{1}{c}{ Variável } & FCO & FCL & LL & LO & $\square \mathrm{Ac}_{\mathrm{cp}}$ \\
\hline FCO & 1 & & & & \\
FCL & $\mathbf{0 , 3 8}$ & 1 & & & \\
LL & $\mathbf{0 , 4 9}$ & $\mathbf{0 , 4 3}$ & 1 & & \\
LO & 0,02 & 0,03 & 0,06 & 1 & \\
$\square \mathrm{Ac}_{\mathrm{cp}}$ & $\mathbf{0 , 6 6}$ & 0,24 & 0,15 & 0,01 & 1 \\
\hline
\end{tabular}

As correlações mais relevantes estão destacadas em negrito na tabela acima. Observe que a variável LO apresenta correlação desprezível (próxima de zero) com as outras quatro variáveis, indicando que os erros de previsão de LO, a partir de FCO, LL e FCO mais $\triangle A c_{c p}$, devem ser bastante elevados, comparativamente aos erros de previsão das demais variáveis. A correlação mais forte é entre $\mathrm{FCO}$ e $\Delta \mathrm{Ac}_{\mathrm{cp}}(0,66)$, justificando o exame das previsões dos fluxos futuros de caixa não somente pelo FCO isolado, mas também verificando se há algum efeito incremental de $\triangle A c_{c p}$ nas previsões.

As correlações de FCO com LL $(0,49)$, de FCO com FCL $(0,38)$ e de FCL com LL $(0,43)$ são relativamente próximas, indicando que não deverá haver diferenças importantes na previsão dos fluxos futuros de FCO, LL e FCL a partir de FCO isolado (ou em conjunto $\left.\operatorname{com} \Delta \mathrm{Ac}_{\mathrm{cp}}\right)$ e de LL.

\section{Testes}

A Tabela 3 mostra os resultados finais dos testes realizados.

Tabela 3 - Projeção de FCO, FCL, LL e LO, Um e Dois Anos à Frente, a Partir de FCO, LL e FCO mais Accruals de Curto Prazo 


\begin{tabular}{|c|c|c|c|c|c|c|c|c|c|c|c|c|}
\hline \multirow{2}{*}{ Modelo } & \multicolumn{3}{|c|}{ FCO } & \multicolumn{3}{|c|}{ FCL } & \multicolumn{3}{|c|}{ LL } & \multicolumn{3}{|c|}{ LO } \\
\hline & DMA & MED & $\mathrm{R}^{2}$ & DMA & MED & $\mathrm{R}^{2}$ & DMA & MED & $\mathrm{R}^{2}$ & DMA & MED & $\mathrm{R}^{2}$ \\
\hline & \multicolumn{12}{|c|}{ Um Ano à Frente } \\
\hline $\mathrm{FCO}$ & 0,162 & 0,001 & 0,354 & 0,120 & 0,014 & 0,240 & 0,115 & 0,032 & 0,505 & 0,681 & $-0,177$ & 0,037 \\
\hline LL & 0,161 & $-0,010$ & 0,478 & 0,125 & $-0,008$ & 0,477 & $0,09 * *$ & 0,011 & 0,725 & 0,689 & $-0,170$ & 0,091 \\
\hline \multirow[t]{2}{*}{$\mathrm{FCO}, \square \mathrm{Ac}_{\mathrm{cp}}$} & $0,160 *$ & 0,012 & 0,466 & 0,118 & 0,014 & 0,441 & 0,115 & 0,031 & 0,617 & 0,686 & $-0,188$ & 0,051 \\
\hline & \multicolumn{12}{|c|}{ Dois Anos à Frente } \\
\hline FCO & 0,171 & $-0,004$ & 0,363 & 0,109 & $-0,011$ & 0,536 & 0,128 & 0,019 & 0,495 & 0,805 & $-0,124$ & 0,022 \\
\hline LL & $0,383 * *$ & $-0,106$ & 0,363 & 0,107 & $-0,059$ & 0,536 & $0,163 * *$ & $-0,021$ & 0,495 & 0,803 & $-0,182$ & 0,018 \\
\hline $\mathrm{FCO}, \square \mathrm{Ac}_{\mathrm{cp}}$ & $0,187^{* *}$ & $-0,002$ & 0,338 & $0,127^{* *}$ & $-0,021$ & 0,289 & 0,12 & 0,017 & 0,673 & 0,811 & $-0,251$ & $-0,003$ \\
\hline
\end{tabular}

${ }^{*}$ ) significativo a $95 \%$, em relação ao modelo $\mathrm{FCO}$ como variável independente; $\left.{ }^{* *}\right)$ significativo a $99 \%$, em relação ao modelo $\mathrm{FCO}$ como variável independente; DMA = Desvio (ou erro) Médio Absoluto. Nos desvios simples em relação à média, os erros negativos compensam os erros positivos, resultando em uma soma dos desvios igual a zero. Para evitar esse problema, optou-se por trabalhar com os desvios absolutos em relação à média, já que o que importa aqui é conhecer o erro médio de previsão dos fluxos futuros, não interessando se este é positivo ou negativo. O DMA equivale à média aritmética dos desvios simples absolutos em relação à média dos erros de previsão de cada variável, de todos os anos. Foi apurada combinando-se a série de erros temporal e transversalmente (pooled-data).

MED = Mediana da série completa de erros de previsão para cada variável. Da série de erros de previsão de cada variável projetada, disposta em pooled-data, foi extraída a Mediana. $\mathrm{R}^{2}$ = coeficiente de determinação (quadrado da correlação) entre o valor real da variável e o valor predito ou projetado para essa mesma variável.

Conforme descrito na seção de metodologia, foram projetados para um e dois anos à frente duas medidas de fluxo de caixa (FCO e FCL) e duas medidas de lucro (LL e LO), a partir do FCO isolado, do LL e do FCO combinado com os accruals de curto prazo $\left(\triangle A c_{c p}\right)$, com o objetivo de verificar se a presença das alocações (accruals), base do regime de competência, torna o Lucro Contábil uma medida superior em relação ao FCO (espécie de lucro por regime de caixa) para projetar os fluxos futuros de caixa e de lucro.

As projeções foram feitas ano a ano para as previsões de um ano à frente, e a cada dois anos para as previsões de dois anos à frente. Este procedimento, denominado tecnicamente de previsão externa à amostra, gera parâmetros de projeção diferentes para cada período (no caso, anual e bianual), pela incorporação gradual dos novos valores periódicos das variáveis à série. Alinha-se à prática possível de quem deseja fazer projeções de variáveis contábeis, que só dispõe das informações existentes até o momento da projeção. Contrasta com um outro tipo de metodologia, ainda encontrada em certas pesquisas e denominada projeção interna à amostra, em que são extraídos parâmetros únicos de projeção a partir da 


\section{repec}

Poder Relativo do Lucro Contábil e do Fluxo de Caixa das operações para prever Fluxos de Caixa

Futuros: um Estudo Empírico no Brasil

amostra completa, que são aplicados período a período nos modelos de previsão.

A Tabela 3 indica que os maiores erros de previsão, um ano e dois anos à frente, ocorreram com a variável Lucro Operacional (LO). Os resultados indicam que durante o período amostral (1996 a 2004) as eventuais tentativas de fazer previsões do Lucro Operacional no Brasil foram, na média, ineficazes. Está-se assumindo, com essa afirmação, que as 92 empresas não-financeiras da amostra representam o "mercado brasileiro" de empresas não- financeiras listadas na Bovespa, uma premissa bastante razoável, pois contempla todas as empresas listadas na Bovespa que apresentaram dados completos para todo o período de nove anos. Os Desvios (erros) Médios Absolutos de previsão (DMA) de LO são bastante elevados. Para um ano à frente, esse erro é de 0,681 quando a variável preditora (variável independente) é o FCO; 0,689 se o preditor for LL; e 0,686 quando se utilizam dois preditores, $\mathrm{FCO}$ e $\triangle \mathrm{Ac}_{\mathrm{cp}}$. Quando a previsão de LO é para dois anos à frente, os erros são ainda maiores, de 0,805, 0,803 e 0,811, respectivamente para FCO, LL e FCO mais $\Delta \mathrm{Ac}_{\mathrm{cp}}$ como preditores. Deve ser ressalvado, contudo, que cinco das 92 empresas da amostra apresentaram desvios bastante elevados, que podem ser interpretados como outliers que puxaram para cima o desvio médio absoluto de LO. Optou-se, porém, em não excluir essas observações, uma vez que essas mesmas empresas permaneceram na análise dos desvios das demais variáveis.

De uma forma geral, observa-se que DMA para dois anos à frente é maior do que para um ano. A exceção ocorre com a previsão de FCL. Nesse caso, a previsão dos Fluxos de Caixa Livres para dois anos à frente, para as variáveis preditoras FCO e LL, revelou-se mais acurada do que para um ano à frente: enquanto FCO e LL não apresentam diferenças significativas nos desvios para prever FCL um ano à frente $(0,120$ e 0,125), há uma redução dos erros de previsão para um horizonte de dois anos à frente, ficando ainda mantida a equivalência de FCO e LL como variáveis preditoras de $\operatorname{FCL}(0,109$ e 0,107).

O Fluxo de Caixa das Operações equipara-se ao Lucro Líquido na previsão de FCO para um ano à frente, conforme pode ser visto, na Tabela 3, pelos seus DMA praticamente iguais $(0,162$ e 0,161 respectivamente). Contudo, FCO é, substancialmente, mais eficaz para prever FCO dois anos à frente (DMA de 0,171) do que LL (DMA de 0,383).

O Lucro Líquido é superior ao Fluxo de Caixa das Operações para prever o Lucro Líquido um ano à frente (DMA de 0,115 para FCO e 0,09 para LL). Contudo, quando se alarga o horizonte de previsão para dois anos à frente, FCO mostra-se, significativamente, superior ao LL para prever LL (DMA de 0,128 para FCO e 0,163 para LL). Este resultado pode decorrer da tendência de reversão dos accruals de curto prazo, documentado no estudo de Sloan (1996), e é consistente com os achados de Lev et al. (2005) para amostra de empresas localizadas nos Estados Unidos.

Um outro aspecto interessante revelado na Tabela 3 é que a adição da variação dos accruals de curto prazo $\left(\Delta \mathrm{Ac}_{\mathrm{cp}}\right)$ ao Fluxo de Caixa das Operações (FCO) não melhorou as previsões dos fluxos futuros, dois anos à frente, de nenhuma das quatro medidas de desempenho 
(FCO, FCL, LL e LO), relativamente ao Fluxo de Caixa das Operações considerado isoladamente (FCO). Ao contrário, o $\mathrm{FCO}$ sozinho é superior ao $\mathrm{FCO}$ e ao $\triangle \mathrm{Ac}_{\mathrm{cp}}$ juntos para prever, dois anos à frente, o próprio FCO (desvios de 0,171 e 0,187, respectivamente) e o FCL (desvios de 0,109 e 0,127, respectivamente). Houve apenas uma discreta melhoria, relativamente ao FCO, na previsão do Fluxo de Caixa das Operações para um ano à frente, uma vez que o desvio médio absoluto do preditor Fluxo de Caixa das Operações em conjunto com os accruals de curto prazo ( $\mathrm{FCO}, \Delta \mathrm{Ac}_{\mathrm{cp}}$ ), de 0,160, é ligeiramente inferior, porém significativo a 95\% de nível de confiança, ao desvio que foi produzido pelo preditor Fluxo de Caixa das Operações isoladamente (FCO), de 0,162. A adição dos accruals de curto prazo $\left(\Delta \mathrm{Ac}_{\mathrm{cp}}\right)$ ao Fluxo de Caixa das Operações (FCO) é, aproximadamente, o capital circulante líquido gerado pelas operações. Pode-se, portanto, concluir que o Fluxo de Caixa das Operações, sozinho, é superior ao capital circulante líquido gerado pelas operações nas previsões dois anos à frente de FCO e FCL.

A análise dos resultados pela Mediana (MED) corrobora os resultados que foram analisados pelos Desvios Médios Absolutos (DMA). De uma forma geral, menores DMAs implicam Medianas mais próximas de zero. Por exemplo, a MED da previsão de FCO para dois anos à frente é de -0,004 para FCO como preditor (DMA de 0,171) e -0,106 (DMA de 0,383 ) para LL como preditor.

Quanto às correlações médias quadradas $\left(R^{2}\right)$ entre as séries de valores preditos e reais para as quatro variáveis, observa-se que a previsão a partir de LL apresenta uma correlação maior com os valores reais das variáveis do que quando a previsão é feita a partir de FCO, mas essas mesmas correlações são praticamente iguais quando o horizonte de previsão passa para dois anos à frente.

Enfim, as principais revelações da Tabela 3 podem ser resumidas como segue:

- As previsões de LO, segundo a definição que a Lei $n^{\circ} 6.404 / 76$ dá para essa variável são pouco eficazes. A inclusão de despesas e receitas financeiras e de equivalência patrimonial muito voláteis em LO, sobretudo para algumas empresas, compromete a acurácia da previsão dessa variável. Sugere-se, em futuros estudos nessa linha, trabalhar com o conceito de Lucro Operacional clássico, o chamado EBIT = Earnings Before Interest and Tax.

- O Lucro Contábil é superior ao Fluxo de Caixa das Operações para prever o Lucro Líquido um ano à frente; mas se o horizonte de previsão aumentar para dois anos, o Fluxo de Caixa das Operações passa a ser superior ao lucro para prever o Lucro Líquido contábil.

- Não há diferenças significativas em se utilizar FCO ou LL para prever os Fluxos de Caixa Futuros, sejam estes o próprio FCO ou o Fluxo de Caixa Livre (FCL), para horizontes de projeção de um e dois anos à frente. A exceção ocorre na previsão de FCO para do Caixa das Operações é superior ao capital circulante líquido gerado pelas operações para prever FCO e FCL dois anos à frente. Na previsão para um ano 


\section{repec}

Poder Relativo do Lucro Contábil e do Fluxo de Caixa das operações para prever Fluxos de Caixa Futuros: um Estudo Empírico no Brasil

à frente, o capital circulante líquido gerado pelas operações é levemente superior ao Fluxo de Caixa das Operações para prever FCO.

\section{CONCLUSÕES}

O FASB estabeleceu, formalmente, em sua estrutura conceitual, há quase 30 anos, que o lucro apurado por regime de competência era superior ao lucro por regime de caixa para projeções de Fluxos de Caixa Futuros. Desde então, uma série de pesquisas foi realizada, sobretudo nos países com forte mercado de capitais, para avaliar empiricamente se era verdadeira a posição assumida pelo FASB.

Embora sem conclusões definitivas, conforme visto na revisão da literatura deste artigo, há uma tendência em se aceitar que o Lucro Contábil é superior ao Fluxo de Caixa das Operações para prever lucros futuros em curto prazo, entendendo-se como curto prazo o período de tempo de até um ano da data da previsão.

A ausência de estudos sobre esse ponto no Brasil motivou a elaboração desta pesquisa. Por meio de uma metodologia de previsão externa à amostra, foram projetadas quatro variáveis de fluxos, sendo duas de fluxo de caixa (Fluxo de Caixa das Operações e Fluxo de Caixa Livre) e duas de lucro (Lucro Líquido e Lucro Operacional), para horizontes de um ano e dois anos à frente do momento de projeção. As projeções foram feitas a partir de três modelos de regressão linear: o primeiro, contendo apenas o Fluxo de Caixa das Operações como variável independente; o segundo, contendo apenas o lucro como variável independente; e o terceiro, contendo o Fluxo de Caixa das Operações e a variação das contas operacionais do Ativo e Passivo Circulantes (accruals de curto prazo) como variáveis independentes.

Constatou-se, na linha das conclusões de alguns estudos estrangeiros, que o Lucro Contábil é significativamente superior ao Fluxo de Caixa das Operações para prever o Lucro Líquido um ano à frente; mas quando o horizonte de projeção muda para dois anos à frente, o Fluxo de Caixa das Operações passa a ser, significativamente, superior ao Lucro Líquido para prever o próprio Lucro Líquido.

Por outro lado, quando se trata de prever o Fluxo de Caixa das Operações, não há diferença significativa em se utilizar o Lucro Contábil ou o Fluxo de Caixa das Operações para estabelecer essas previsões para um ano à frente. Contudo, consistente com os resultados do estudo de Lev, Li e Sougiannis (2005), no mercado americano, nas previsões de dois anos à frente o Fluxo de Caixa das Operações revelou-se, significativamente, superior ao Lucro Contábil para prever o Fluxo de Caixa das Operações.

Esta pesquisa revela também, indiretamente, que a medida de Lucro Operacional definida na Lei $n^{\circ}$ 6.404/76 parece inadequada. A inclusão das despesas financeiras e do resultado de equivalência patrimonial no grupamento do resultado operacional, como é feito no 
Brasil, torna essa medida de difícil previsão, em razão da alta volatilidade desses elementos. Para todas as variáveis utilizadas como preditoras do Lucro Operacional nesse estudo, os erros de previsão foram excessivamente elevados comparativamente aos desvios verificados na previsão das demais variáveis. Ressalve-se, entretanto, que a alta volatilidade ocorreu durante o período amostral desta pesquisa (1996 a 2004), sobretudo para algumas empresas. A extrapolação dessa constatação para outros períodos deve ser feita com reservas.

A utilização conjunta do Fluxo de Caixa das Operações e da variação das contas operacionais de curto prazo ( $\left.\mathrm{FCO}, \Delta \mathrm{Ac}_{\mathrm{cp}}\right)$ no modelo de previsão não melhorou, significativamente, a previsão que foi obtida, utilizando-se apenas o Fluxo de Caixa das Operações como variável preditora dos fluxos futuros. Ao contrário, nas previsões de um ano à frente de FCO e FCL, o Fluxo de Caixa das Operações revelou-se superior, como preditor, ào $\mathrm{FCO}$ e ao $\triangle \mathrm{Ac}_{\mathrm{cp}}$ juntos. Esta é uma outra conclusão interessante, pois revela indiretamente que o capital circulante líquido gerado pelas operações (FCO mais variação dos accruals de curto prazo) não é uma medida superior ao FCO para prever fluxos futuros.

A previsão dos fluxos futuros foi feita regredindo-se transversalmente (cross-section) a variável projetada em $t$ contra a variável independente em $t-1$ (modelo de um ano à frente) e em $t$-2 (modelo de dois anos à frente). Os parâmetros dos modelos de estimativas (alfa e beta) assim obtidos foram multiplicados pelos valores reais da variável independente em $t$ a fim de obterem-se as previsões de FCO, FCL, LL e LO em $t+1$ (modelo de um ano à frente) e em $t+2$ (modelo de dois anos à frente). Sugere-se que sejam feitas outras pesquisas nessa mesma linha com o uso de outras metodologias de projeção (random walk, séries temporais, etc.) e também com previsões para horizontes mais longos, por exemplo, três, quatro anos à frente.

Os resultados desta pesquisa deixam uma importante questão em aberto para reflexão. O processo de alocação de custos expirados ao resultado é um dos principais pilares do modelo de contabilidade financeira em todo o mundo. No entanto, esse modelo não parece ser superior ao lucro por regime de caixa na previsão dos fluxos livres de caixa futuros, que é a medida de valor que fundamentalmente interessa a quem decide investir na empresa. Vale a pena insistir nesse modelo de relevância duvidosa, ou deve-se caminhar, progressivamente, para um outro modelo contábil, fundamentado na idéia de valor, como já vem sendo feito para os instrumentos financeiros?

\section{REFERÊNCIAS}

Ali, A. The Incremental Information Content of Earnings, Working Capital from Operations, and Cash Flows. Journal of Accounting Research, 32, 1994, 61-74.

Barth, M., Beaver, W. H., Hand, J. R. M. e Landsman, W. R. Accruals, Accounting-Based Valuation Models, and the Prediction of Equity Values. Working Paper, Stanford University of North Carolina at Chapel Hill, 2005. 
Poder Relativo do Lucro Contábil e do Fluxo de Caixa das operações para prever Fluxos de Caixa Futuros: um Estudo Empírico no Brasil

Barth, M., Cram, D. e Nelson, K. Accruals and the Prediction of Future Cash Flows. The Accounting Review, 76, 2001, 27-58.

Bernard, V. L. e Stober, T. L. The Nature and Amount of Information in Cash Flows and Accruals. The Accounting Review, 1989, 624-652.

Bowen, R., Burgstahler, M. D. e Daley, L. A. The Incremental Information Content of Accruals Versus Cash Flows. The Accounting Review, 1987, 723-747.

Bujaki, M. L. e Richardson, A. J. A Citation Trail Review of the Uses of Firm Size in Accounting Research. Journal of Accounting Literature, 1997.

Dantas, J. A. Reação do Mercado à Alavancagem Operacional: Um Estudo Empírico no Brasil. Dissertação (Programa Multiinstitucional e Inter-regional de Mestrado em Ciências Contábeis, UnB, UFPB, UFPE e UFRN), Universidade de Brasília, 2005.

Dechow, P. Accounting Earnings and Cash Flows as Measures of Firm Performance: The Role of Accruals. Journal of Accounting and Economics, 1996, 18, 3-42.

FASB - Financial Accounting Standards Board. FAS-95 - Statement of Cash Flows. www. fasb.org. Consulta em 6/2/2006.

Finger, C. The Ability of Earnings to Predict Future Earnings and Cash Flows. Journal of Accounting Research, 1994, 32, 210-223.

Goyal, A. e Welch, I. A Comprehensive Look at the Empirical Performance of Equity Premium Prediction. Yale University, Working Paper, 2004.

Guerreiro, R. Sistema de Informação de Gestão Econômica: Uma Contribuição à Teoria da Comunicação em Contabilidade. Tese (Doutorado em Contabilidade e Controladoria), Departamento de Contabilidade e Atuária, FEA/USP, 1989.

Ijiri, Y. Cash is a Fact, but Income is a Forecast. Working Paper, Carnegie Melon University, 2002.

Lev, B., Li, S. e Sougiannis, T. Accounting Estimates: Pervasive, Yet of Questionable Usefulness. Working Paper, New York University, New York, abril 2005.

Lopes, A. B. A Relevância da Informação Contábil para o Mercado de Capitais: o Modelo de Ohlson Aplicado à Bovespa. Tese (Doutorado em Contabilidade e Controladoria), Departamento de Contabilidade e Atuária, FEA/USP, 2001.

Lubberink, M. e Pope, P. Does Scale Make a Difference? Working Paper. www.ssrn.com, 2005. 
Lustosa, P. R. B. Um Estudo das Relações entre o Lucro Contábil, os Fluxos Realizados de Caixa das Operações e o Valor Econômico da Empresa: Uma Simulação Aplicada a um Banco Comercial. Tese (Doutorado em Contabilidade e Controladoria), Departamento de Contabilidade e Atuária, FEA/USP, 2001.

Martinez, A. L. Gerenciamento dos Resultados Contábeis: Estudo Empírico das Companhias Abertas Brasileiras. Tese (Doutorado em Contabilidade e Controladoria), Departamento de Contabilidade e Atuária, FEA/USP, 2002.

Martins, E. Avaliação de Empresas: Da Mensuração Contábil à Econômica. São Paulo: Atlas, 2001.

Pfeiffer, R. J., Elgers, P. T., Lo, M. H. e Rees, L. L. Additional Evidence on the Incremental Information Content of Cash Flows and Accruals: The Impact of Errors in Measuring Market Expectations. The Accounting Review, 73, 1998, 373-385.

Poon, S. e Granger, C. Forecasting Volatility in Financial Markets. Journal of Economic Literature, $\mathrm{XLI}, 478-539$.

Rayburn, J. D. The Association of Operating Cash Flows and Accruals with Security Returns. Journal of Accounting Research, 1986, 112-133.

Sloan, R. G. Do Stock Prices Fully Reflect Information in Accruals and Cash Flows About Future Earnings? The Accounting Review, 71, 1996, 289-315.

Wilson, G. P. The Relative Information Content of Accruals and Cash Flows: Combined Evidence at the Earnings Announcement and Annual Report Release Date. Journal of Accounting Research, 1986, 165-200. 